\title{
Editorial: Endophenotypes for Schizophrenia and Mood Disorders: Implications from Genetic, Biochemical, Cognitive, Behavioral, and Neuroimaging Studies
}

\author{
Błażej Misiak ${ }^{1,2 *}$, Dorota Frydecka ${ }^{1}$ and Janusz K. Rybakowski ${ }^{3}$ \\ ${ }^{1}$ Department of Psychiatry, Wroclaw Medical University, Wroclaw, Poland, ${ }^{2}$ Department of Genetics, Wroclaw Medical \\ University, Wroclaw, Poland, ${ }^{3}$ Department of Adult Psychiatry, Poznan University of Medical Sciences, Poznan, Poland
}

Keywords: endophenotype, schizophrenia, mood disorders, psychosis, genetics

The Editorial on the Research Topic

Endophenotypes for schizophrenia and mood disorders: implications from genetic, biochemical, cognitive, behavioral, and neuroimaging studies

OPEN ACCESS

Edited by:

Ripu D. Jindal,

University of Alabama at Birmingham,

USA

Reviewed by:

Michele Fornaro,

Columbia University, USA

${ }^{*}$ Correspondence:

Błażej Misiak

mblazej@interia.eu

Specialty section:

This article was submitted

to Affective Disorders and

Psychosomatic Research,

a section of the journal

Frontiers in Psychiatry

Received: 29 March 2016

Accepted: 28 April 2016

Published: 11 May 2016

Citation:

Misiak B, Frydecka D and

Rybakowski JK (2016) Editorial:

Endophenotypes for Schizophrenia

and Mood Disorders: Implications

from Genetic, Biochemical, Cognitive,

Behavioral, and Neuroimaging

Studies.

Front. Psychiatry 7:83.

doi: 10.3389/fpsyt.2016.00083
Schizophrenia and mood disorders represent complex phenotypes that are characterized by multidimensional psychopathology and biological underpinnings. After many years of clinical observations, it has been recognized that there are several intermediate phenotypes between schizophrenia and mood disorders that share common clinical characteristics. This observation has provided grounds for the concept of endophenotypes, crossing traditional diagnostic boundaries and opening perspectives for better understanding of mental disorders. Indeed, the term endophenotype refers to a measurable construct that bridges a gap between phenotype expression and genetic variability. This intermediate phenotype construct must meet four characteristics: the association with illness in the population, heritability, state-independent manifestation (expression of the phenotype regardless of the illness activity), and cosegregation with illness in families (1). Consequently, a number of promising genetic, biochemical, cognitive, behavioral, and neuroimaging endophenotypes emerged providing a broader insight into the nature of schizophrenia and mood disorders.

In this research topic, a few promising candidates of clinical, biochemical, and neuroimaging endophenotypes have been proposed. Grant describes various aspects of schizotypy and proposes that schizotypy itself might be a suitable endophenotype of schizophrenia. Following the definition of endophenotype developed by Gottesman and Gould (1), the author indicates that schizophrenia patients have high levels of schizotypy that are even higher than in other psychotic disorders. Grant also provides evidence that schizotypy is highly heritable and cosegregates with schizophrenia with the illness in families. Additionally, it has been reported that healthy relatives of schizophrenia patients have higher levels of schizotypy than healthy individuals with negative family history of schizophrenia. Finally, Grant shows that schizotypy is a stable trait with high test-retest reliability in patients with schizophrenia. However, it should be noted that higher levels of schizotypy have been also reported in patients with bipolar disorder (BD) (2-4). Therefore, future studies should focus on determining the validity of schizotypy together with its specific dimensions as an endophenotype of schizophrenia or BD.

In two studies, the role of structural and functional brain alterations as potential endophenotypes of psychosis dimension has been investigated. In the large and multisite bipolar-schizophrenia network on intermediate phenotypes (B-SNIP) study, Wang et al. found that functional alterations in 
a prefrontal-striatal-thalamic-cerebellar network and structural disturbances in the default mode network are common abnormalities across various psychotic disorders. Based on the data from B-SNIP study, Soh et al. performed joint independent component analysis of awake EEG frequency activity and MRI gray matter volumes in patients with psychotic disorders. Authors found a single component differentiating schizophrenia patients and healthy individuals. Specifically, they reported increased posterior alpha activity associated with decreased volume in inferior parietal lobe, supramarginal, parahippocampal gyrus, middle frontal, inferior temporal gyri, and increased volume of uncus and culmen in patients with schizophrenia.

In the last article, Kim et al. in their mini review proposed that phospholipase C- $\beta 1$ (PLC- $\beta 1$ ) hypofunction might be an endophenotype of schizophrenia. This enzyme is involved in phosphoinositide signaling, which is one of the main G-protein-related pathways in the central nervous system. It has been reported that PLC- $\beta 1^{-/-}$mice express several negative-like symptoms and cognitive deficits that resemble those observed in schizophrenia $(5-7)$. In addition, lower PLC- $\beta 1$ has been found in several brain regions of patients with schizophrenia (8). Interestingly, the role of aberrant phospholipase C- $\gamma 1$ (PLC- $\gamma 1$ ) signaling has been also implicated in the pathophysiology of BD [for review, see Ref. (9)). In addition, polymorphism of the PLC- $\gamma 1$ gene has been found to predict response to lithium treatment (10). A recent analysis of data from the genome-wide association study (GWAS) also revealed that genetic variation in phospholipase $\mathrm{C}$ signaling might be associated with a risk of $\mathrm{BD}$ (11). Therefore, more research is needed to disentangle the role of impaired phospholipase $\mathrm{C}$ signaling as an endophenotype for both schizophrenia and BD.

Although a number of candidate endophenotypes for schizophrenia and psychotic disorders have been proposed in this research topic, studies replicating these findings in large

\section{REFERENCES}

1. Gottesman II, Gould TD. The endophenotype concept in psychiatry: etymology and strategic intentions. Am J Psychiatry (2003) 160:636-45. doi:10.1176/ appi.ajp.160.4.636

2. Heron J, Jones I, Williams J, Owen MJ, Craddock N, Jones LA. Self-reported schizotypy and bipolar disorder: demonstration of a lack of specificity of the Kings Schizotypy Questionnaire. Schizophr Res (2003) 65:153-8. doi:10.1016/ S0920-9964(03)00004-5

3. Dembińska-Krajewska D, Rybakowski J. The concept of schizotypy and significance of the Oxford-Liverpool Inventory of Feelings and Experiences (O-LIFE). Neuropsychiatr Neuropsychol (2014) 9:4-13.

4. Brosey E, Woodward ND. Schizotypy and clinical symptoms, cognitive function, and quality of life in individuals with a psychotic disorder. Schizophr Res (2015) 166:92-7. doi:10.1016/j.schres.2015.04.038

5. Koh HY, Kim D, Lee J, Lee S, Shin HS. Deficits in social behavior and sensorimotor gating in mice lacking phospholipase Cbeta1. Genes Brain Behav (2008) 7:120-8. doi:10.1111/j.1601-183X.2007.00351.x

6. McOmish CE, Burrows E, Howard M, Scarr E, Kim D, Shin HS, et al. Phospholipase C-betal knockout mice exhibit endophenotypes modeling schizophrenia which are rescued by environmental enrichment and clozapine administration. Mol Psychiatry (2008) 13:661-72. doi:10.1038/sj.mp.4002046

7. Kim SW, Seo M, Kim DS, Kang M, Kim YS, Koh HY, et al. Knockdown of phospholipase C-betal in the medial prefrontal cortex of male mice impairs working memory among multiple schizophrenia endophenotypes.J Psychiatry Neurosci (2015) 40:78-88. doi:10.1503/jpn.130285 samples are required. In addition, future studies must look at these findings in a broader context of endophenotype criteria and verify how well they suit their original purpose - linking less complex disease phenotype to its genetic underpinnings. It is important to note that studies investigating endophenotypes should take into account that psychosis dimension is itself a heterogeneous construct. Indeed, psychotic symptoms associated with schizophrenia have different features compared to those observed in BD. For instance, most psychotic features associated with manic episodes tend to have a different, usually self-limited and self-remitting course. These symptoms appear in most severe episodes of BD. On the contrary, psychotic symptoms in schizophrenia have a more long-lasting course. This observation give rise to the conclusion that more objective and reliable endophenotypes offer a prospect of defining more etiologically homogenous subgroups of human psychopathology and thus facilitate our understanding of the basis and causal pathways of mental disorders. Finally, the existence of relevant endophenotypes should initiate discussion about reappraisal of the research diagnostic criteria beyond traditional diagnostic systems to enable better understanding of mechanisms underlying schizophrenia and mood disorders.

\section{AUTHOR CONTRIBUTIONS}

All author listed have made substantial, direct, and intellectual contribution to the work and approved it for publication.

\section{ACKNOWLEDGMENTS}

Editors are deeply grateful to all authors and reviewers, who contributed to this research topic. BM is supported by the START 2015 scholarship provided by the Foundation for Polish Science.

8. du Bois TM, Deng C, Huang XF. Membrane phospholipid composition, alterations in neurotransmitter systems and schizophrenia. Prog Neuropsychopharmacol Biol Psychiatry (2005) 29:878-88. doi:10.1016/j. pnpbp.2005.04.034

9. Yang YR, Kang DS, Lee C, Seok H, Follo MY, Cocco L, et al. Primary phospholipase C and brain disorders. Adv Biol Regul (2015). doi:10.1016/j. jbior.2015.11.003

10. Turecki G, Grof P, Cavazzoni P, Duffy A, Grof E, Ahrens B, et al. Evidence for a role of phospholipase C-gammal in the pathogenesis of bipolar disorder. $\mathrm{Mol}$ Psychiatry (1998) 3:534-8. doi:10.1038/sj.mp.4000447

11. Nurnberger JI Jr, Koller DL, Jung J, Edenberg HJ, Foroud T, Guella I, et al. Identification of pathways for bipolar disorder: a meta-analysis. JAMA Psychiatry (2014) 71:657-64. doi:10.1001/jamapsychiatry.2014.176

Conflict of Interest Statement: The authors declare that the research was conducted in the absence of any commercial or financial relationships that could be construed as a potential conflict of interest.

Copyright (c) 2016 Misiak, Frydecka and Rybakowski. This is an open-access article distributed under the terms of the Creative Commons Attribution License (CC BY). The use, distribution or reproduction in other forums is permitted, provided the original author(s) or licensor are credited and that the original publication in this journal is cited, in accordance with accepted academic practice. No use, distribution or reproduction is permitted which does not comply with these terms. 